\title{
The distance to the solar wind termination shock in 1993 and 1994 from observations of anomalous cosmic rays
}

\author{
E. C. Stone and A. C. Cummings \\ Space Radiation Laboratory, California Institute of Technology, Pasadena \\ W. R. Webber \\ Department of Astronomy, New Mexico State University, Las Cruces
}

\begin{abstract}
Using data from the Voyager and Pioneer spacecraft, we examine the energy spectra of anomalous cosmic rays (ACRs) during 1992-1994, a period of rapidly increasing intensities of these particles in the outer heliosphere. The 1992 period appears to be in nonequilibrium, marked by a rapid decrease in the tilt of the current sheet from $\sim 63^{\circ}$ to $\sim 35^{\circ}$ and a complex, evolving magnetic topology that affects interplanetary drifts and drift acceleration along the solar wind termination shock. In addition, large propagating interplanetary disturbances in 1991 may have weakened the shock temporarily, reducing the intensity of accelerated ions. The 1993-1994 period appears to be in quasi-equilibrium, marked by a stable tilt of the current sheet and a steady, large-scale magnetic topology that provides stable conditions for propagation and acceleration. The ACR intensities of higher-rigidity particles rise slowly during this period, suggesting that the shock intensity has stabilized. At lower rigidities the ACR intensities are increasing rapidly, which we attribute to decreasing modulation. During 1994 days 157-209, we cstimate that the shock was at $85 \pm 5 \mathrm{AU}$. The shock strength $s$ is estimated to be $2.63 \pm 0.14$, significantly weaker than a strong shock $(s=4)$ and consistent with a termination shock modified by the pressure of galactic cosmic rays. By comparing these 1994 observations with those made in 1987, we estimate that the latitudinal gradient of ACR He with energies 31-57 $\mathrm{MeV}$ nucleon ${ }^{-1}$ is $\sim 1.9 \% / \mathrm{deg}$ along the shock from the equator to the pole. We estimate that the ratio of the efficiency of injection of $\mathrm{He}^{+}$to $\mathrm{H}^{+}$pickup ions into the acceleration process is $7.0+1.6$.
\end{abstract}

\section{Introduction}

Anomalous cosmic rays (ACRs) are thought to be interstellar neutrals [Fisk et al., 1974] that have become ionized in the interplanetary medium and accelerated in the outer heliosphere, probably at the solar wind termination shock [Pesses et $a l ., 1981]$. Seven elements have been identified in the ACR component: H, He, C, N, O, Ne, and Ar [McDonald et al., 1974; Garcia-Murioz et al., 1973; Hovestadl et al., 1973; Currorrings and Stone, 1988, 1990; Christian et al., 1988, 1995; McDonald et al., 1995]. Recently, observations have confirmed that the ACRs are singly ionized [Adams et al., 1991; Singh et al., 1991; Klecker et al., 1995], and a study of their gradients has suggested that the solar wind termination shock was at $67 \pm 5 \mathrm{AU}$ in 1987 during the last period of minimum solar activity [Cummings et al., 1994].

Other recent estimates of the location of the termination shock based on dynamic prcssurc balance [Belcher et al., 1993; Whang and Burlaga, 1993; Lallement et al., 1993], hydrogen Lyman $\alpha$ resonant scattering [Hall et al., 1993], and kilohertz radio emissions [Grzedielski and Lazarus, 1993; Zank et al., 1994] range from 60 to 105 AU. However, Gurnett et al. [1993] ascribe the radio emissions to the heliopause region located at 116 to 177 AU.

Copyright 1996 by the American Geophysical Union.

Paper number 96JA00413.

0148-0227/96/96JA-00413\$09.00
Cummings et al. [1994] based their estimate of the location of the termination shock in 1987 on a model in which positive particles were drifting into the inner heliosphere along the Sun's neutral sheet. The radial gradient of the ACRs was obscrved to be correlated with the tilt of the ncutral shcet for tilts less than $\sim 30^{\circ}$. The estimate of $67 \mathrm{AU}$ for the shock location was derived by using the observed tilt of the sheet to infer the local gradient of the particles and then extrapolating the ACR intensity outward from the position of the Voyager 2 (V2) and Pioneer 10 (P10) spacecraft to a location where the extrapolated intensity was steady with time.

During the current segment of the solar cycle, after the solar magnetic field reversed in 1990, the ACR particles are expected to drift downward onto the heliographic equator from the polar regions [Jokipii et al., 1977]. The cosmic ray intensity is expected to be less responsive to the changes in the tilt angle than in the $A<0$ period [Le Roux and Potgieter, 1990], and hence we do not use the same technique of extrapolation to the shock that was employed for the 1987 data.

In the current study we have used another technique, similar to that used by I $[1990$ ] for estimating the location of the solar wind termination shock, that is valid for $\sim 1993$ onward during periods without large transient disturbances such as global merged interaction regions (GMIRs). To estimate the location of the solar wind termination shock, we employ a spherically symmetric model of solar modulation. Preliminary accounts of this work have been presented at the 24 th International Cosmic Ray Conference [Stone et al., 1995a, b]. 


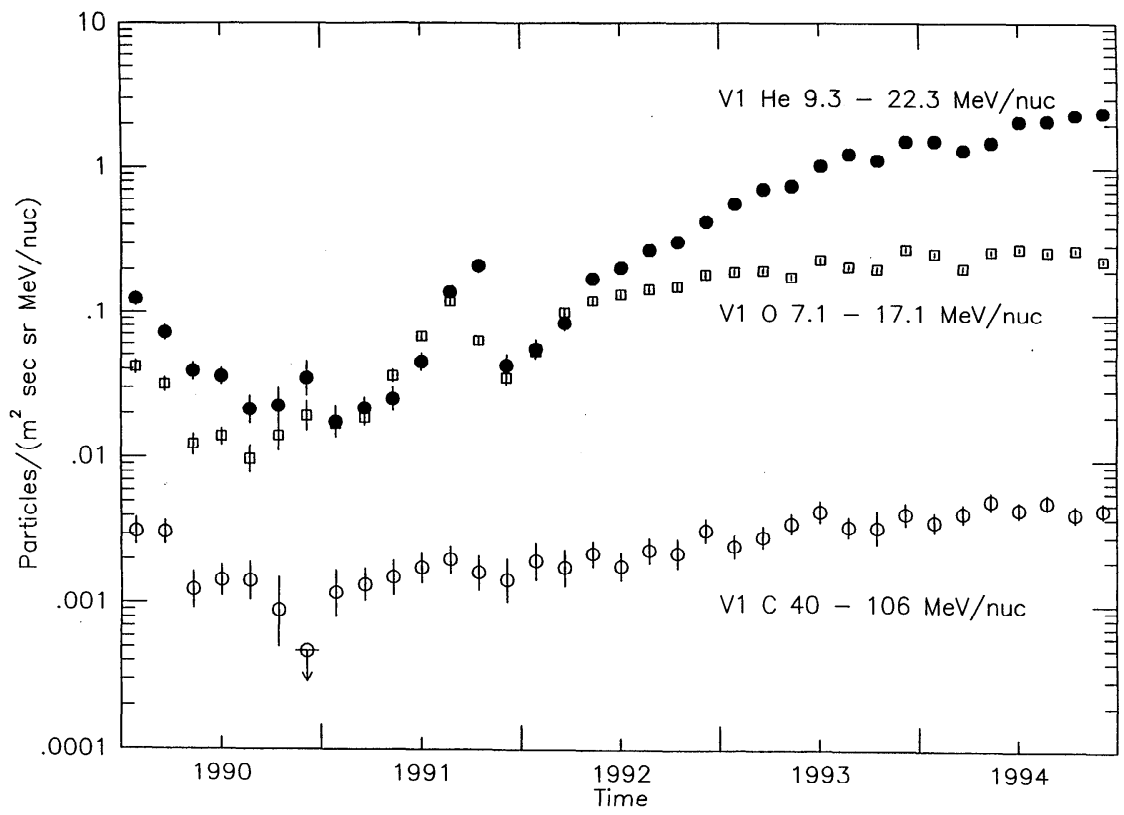

Figure 1. Intensities of cosmic rays measured at Voyager 1. Solid circles represent helium with 9.3-22.3 $\mathrm{MeV}$ nucleon ${ }^{1}$ ( 0.53 to $\left.0.82 \mathrm{GV}\right)$; open squares are oxygen with 7.1-17.1 MeV nucleon ${ }^{-1}$ (1.84 to $2.87 \mathrm{GV}$ ); and open circles are carbon with $40-106 \mathrm{MeV}^{\text {nucleon }}{ }^{-1}$ (0.55 to $\left.0.91 \mathrm{GV}\right)$.

\section{Observations}

In Figure 1 we show the time histories of the intensity of $\mathrm{He}$, $\mathrm{O}$, and $\mathrm{C}$ observed with the cosmic-ray experiment on Voyager 1 (V1) [Stone et al., 1977]. The carbon intensity is for the energy range $40-106 \mathrm{MeV}^{\text {nucleon }}{ }^{-1}$ and is entirely due to galactic cosmic rays which are fully ionized. Prior to 1992 the $\mathrm{He}$ intensity is a mixture of solar, galactic, and anomalous cosmic rays. By 1992 the anomalous cosmic rays dominate this intensity. The $\mathrm{O}$ intensity is dominated by anomalous cosmic rays throughout the period shown in Figure 1.

The $\mathrm{V} 1 \mathrm{He}$ and $\mathrm{O}$ intensity profiles show the result of intense solar activity in early 1991. The ACR intensities rise rapidly after the effects of the transient decrease have passed. From the beginning of 1992 to the end of 1994, the ACR He intensity shows a $\sim 40$-fold increase and the ACR O intensity increases by a factor of $\sim 3$. Most of the $O$ increase occurs during 1992, whereas the $\mathrm{He}$ intensity continues to increase steadily to the end of 1994 . Following a small transient decrease in late 1991, the galactic cosmic ray $\mathrm{C}$ intensity rises by a factor of $\sim 2.5$ from the beginning of 1992 to the end of 1994 . The energy interval of the $\mathrm{O}$ intensity measurements corresponds to a mean rigidity of $\sim 2.4 \mathrm{GV}$, whereas the He intensity measurements correspond to a lower rigidity, $-0.7 \mathrm{GV}$. The equally rapid increases of the $0.7 \mathrm{GV}$ and the $2.4 \mathrm{GV}$ intensities in 1992 suggest a period of nonequilibrium recovery following the transient in late 1991. The much smaller increase in the $2.4 \mathrm{GV}$ intensity during 1993 and 1994 suggests a period of quasi-equilibrium, during which the high-rigidity intensity is nearly the same as the unmodulated shock intensity and the lower-rigidity intensity continues to increase in response to decreasing modulation.

In Figure 2 we show the time dependence of several parameters of interest to this study. Figure $2 \mathrm{c}$ shows the radial gradient of the 10 to $22 \mathrm{MeV}$ nucleon ${ }^{-1} \mathrm{He}$ intensities using the three-spacecraft method. In this method, we assume a constant radial and latitudinal gradient in each 52-day period and solve for cach from the two measured intensity ratios $j_{P 10} / j_{V 2}$ and $j_{V 1} / j_{V 2}$, where $j_{P 10}, j_{V 2}$, and $j_{V 1}$ are the intensities at P10, V2, and V1, respectively (see Cummings et al. [1987] for more explanation). The radial gradient in Figure $2 c$ shows no systematic trend but does exhibit nonstatistical variations.

In Figure 2e we show the tilt of the neutral sheet [Hoeksema, 1992] shifted to the midway position between V2 and P10. The tilt is in a rapid, steady decline until approximately the beginning of 1993 , at which time it levels off at $-35^{\circ}$. The rapidly changing tilt in 1992 implies that the magnetic field topology in the heliosphere is also evolving in a complex way, since the Sun's magnetic field is embedded in the solar wind and carried with it into the outer heliosphere. This changing magnetic field topology will affect the drift motion of the particles as well as the drift acceleration of the ACR particles at the solar wind termination shock. In addition, we might expect the GMIR [McDonald et al., 1994] that evolved from the 1991 solar activity to cause other complex changes in the magnetic field topology and in the acceleration conditions at the shock [Zank et al., 1994]. An interaction of this structure with the heliopause is thought to be responsible for the low-frequency radio emissions observed in 1992 and 1993 by the plasma wave experiment on the Voyager spacecraft [Gurnett et al., 1993].

In Figure 3 we show the energy spectrum of $\mathrm{He}$ at V1 for seven 52-day periods beginning with days 105-157, 1992, and ending with 313-365, 1994. For the two 1992 spectra the entire energy spectrum is growing in intensity uniformly over the full energy range shown ( $\sim 3$ to $60 \mathrm{MeV}$ nucleon $\left.^{-1}\right)$. This is consistent with a nonequilibrium period caused by effects on the drift of the particles from the changing magnetic field topology (Figure 2e) and effects of the 1991 GMIR on the strength of the termination shock. Beginning in 1993 the high-energy end of the spectrum changes intensity only slowly, whereas the lower-energy end continues to rise rapidly. This is consistent with the onset of a quasi-equilibrium period with a stable magnetic field topology (see Figure 2e) giving rise to stable 
conditions for acceleration and propagation. The continued increase in intensities at low energies could result from an energy-dependent mean free path that is increasing with time or a decreasing distance between the shock and V1. The intensity increase is smaller at high energies where the modulation between the shock and the spacecraft is small. We note that the energy of the peak intensity of ACR He is $\sim 6 \mathrm{MeV}$ nucleon $^{-1}$ in late 1994 , lower than previous observations and consistent with a lower level of ACR modulation than at any lime in the past.

\section{Distance to the Shock and Shock Spectrum}

In order to estimate the distance to the termination shock, we have compared our observations at V1 and V2 during the quasi-equilibrium period in 1993 and 1994 with a spherically symmetric equilibrium model of the propagation of ACRs. In
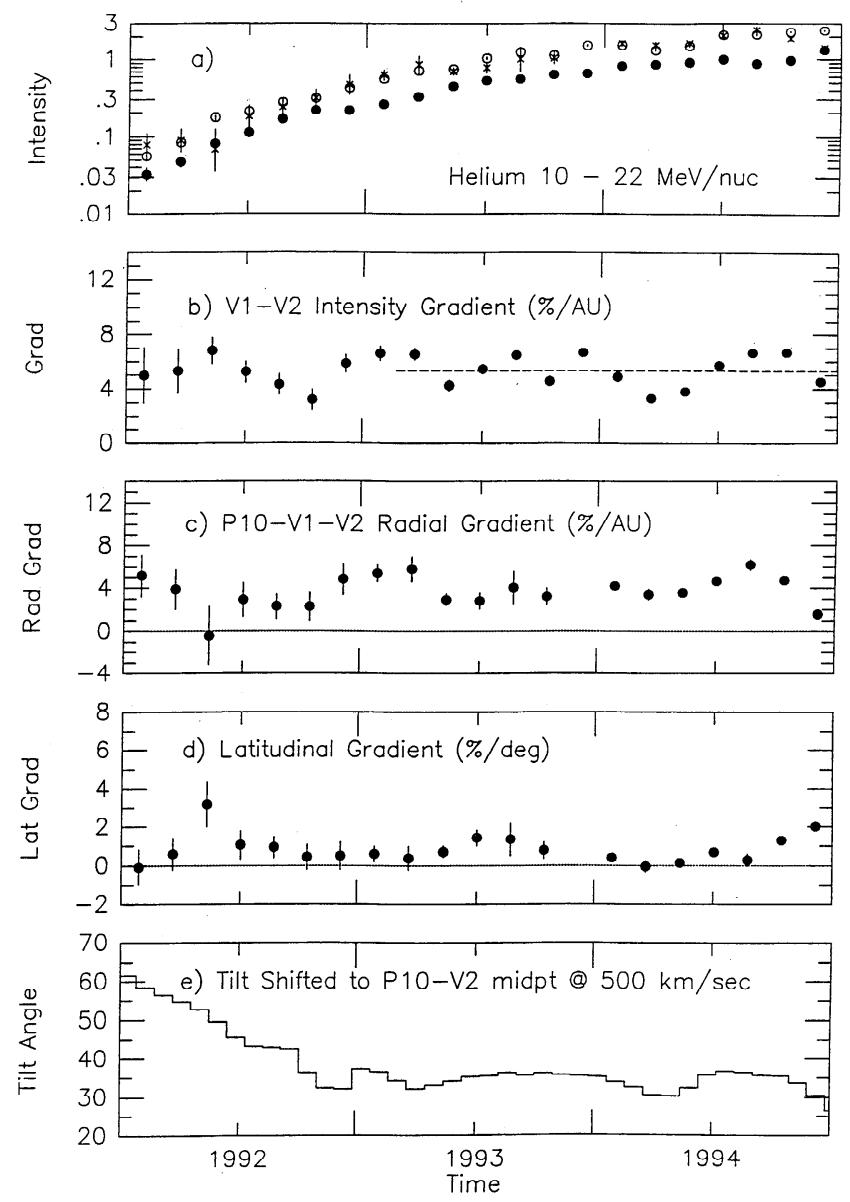

Figure 2. (a) Intensity of helium (52-day averages) measured at P10 (crosses), V2 (solid circles), and V1 (open circles) versus time. The dashed line is the mean for the period indicated. (b) Intensity gradient between V1 and V2 for He with 9.3-22.3 MeV nucleon ${ }^{-1}$. (c) Radial gradient of ACR He with $\sim 10-22$ $\mathrm{MeV}$ nucleon $^{-1}$. (d) Latitudinal gradient of ACR He with $\sim 10-22 \mathrm{MeV}^{\text {nucleon }}{ }^{-1}$. (e) Estimated tilt of the neutral current sheet shifted to the midpoint of V2 and P10. Each tilt observation (J. T. Hoeksema, private communication, 1994) covers a single solar rotation or $\sim 26$ days. We have performed a 3-solar-rotation moving average on the supplied tilt data set before plotting in order to approximate the average conditions between V2 and P10, which are $~ 17$ AU apart.

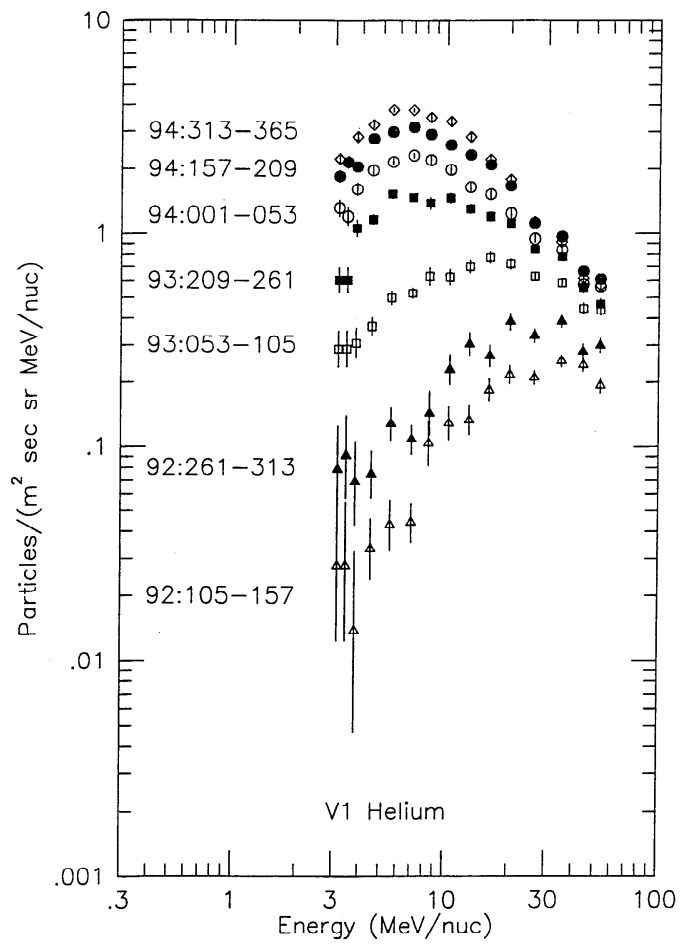

Figure 3. Energy spectrum of helium measured at V1 for seven time periods.

1987 there were large latitudinal gradients of ACRs which would have made meaningful comparison with a spherically symmetric model difficult. Figure 2a shows 52-day average intensities of helium observed on V1, V2, and P10 with energies $\sim 10-22 \mathrm{MeV}$ nucleon $^{-1}$ during 1992-1994. From the shape of the energy spectra we believe that the intensity in this energy interval is dominated by ACRs throughout the period shown, although the first two periods in 1992 have substantial contributions from galactic cosmic rays (GCRs). The intensities at all three spacecraft increase by a factor of $\sim 40$ from the beginning of 1992 to the end of 1994 . The P10 and V1 intensities are in approximate agreement, although P10 leads V1 by a distance which varies from 6.2 to 4.2 AU during this period, and we would expect that the P10 intensity would be somewhat larger than the intensity at V1 if spherical symmetry prevails. The intensity at V2 is consistently lower than the intensity at P10 and V1.

In Figure 2d we show the latitudinal gradients, which show no systematic trend and the mean is $0.78 \% \mathrm{deg}^{-1}$ or cquivalently $\sim 0.8 \% \mathrm{AU}^{-1}$ in the latitudinal direction as compared with $\sim 4 \% \mathrm{AU}^{-1}$ in the radial direction (see Figure 2c). Although the intensity gradient is mainly radial and therefore resembles that of a spherically symmetric model, the small positive latitudinal gradient means that the spectrum observed out of the heliographic equatorial plane at a given radius corresponds to that which would be observed at a somewhat larger "effective radius" in the equatorial plane. We use the following equation to estimate the effective location of a particular spacecraft:

$$
\ln \left(r_{\text {eff }} / r_{\text {act }}\right)=\left(G_{\Theta} \cdot \Theta / G_{0}\right)
$$

where $G_{\Theta}$ is the average latitudinal gradient and $r_{\text {efr }}$ is the effective location, $r_{\text {act }}$ is the actual location, and $\Theta$ is the latitude of the spacecraft, and where we have used a model in 
Table 1. Spacecraft Locations

\begin{tabular}{ccccc}
\hline Pcriod & Spacecraft & $\begin{array}{c}\text { Latitude, } \\
\text { deg }\end{array}$ & $\begin{array}{c}\text { Actual } \\
\text { Radius, } \\
\text { AU }\end{array}$ & $\begin{array}{c}\text { Effective } \\
\text { Radius, } \\
\text { AU }\end{array}$ \\
\hline Days 157-209, & V1 & 32.5 & 56.3 & 64.0 \\
1994 & V2 & -12.0 & 43.3 & 45.4 \\
Days 157-209, & V1 & 32.2 & 52.6 & 59.8 \\
1993 & V2 & -9.5 & 40.4 & 42.0 \\
\hline
\end{tabular}

which the radial gradient in the intensity $j$ is proportional to $1 / r$, with $(1 / j)(\partial j / \partial r)=G_{0} / r$. In this work we assume that the particle distributions are symmetrical about the heliographic equator [Jokipii, 1990], so we use the absolute value of the spacecraft latitude in all cases.

We thus need to know the average latitudinal gradient $G_{\Theta}$ and the average radial gradient parameter $G_{0} . G_{0}$ is related to the three-spacecraft radial gradient $G_{r}$ by

$$
G_{0}=\frac{\Delta r \cdot G_{r}}{\ln \left(r_{10} / r_{2}\right)}
$$

where $r_{10}$ and $r_{2}$ are the radial positions of $\mathrm{P} 10$ and $\mathrm{V} 2$, respectively, and $\Delta r$ is the radial separation distance between them.

It is important to pick time periods for study in which transient disturbances at V1 and V2 are absent. In Figure 2b we show the gradient of the intensities from V1 to V2:

$$
G_{V 1 V 2}=\ln \left(j_{V 1} / j_{V 2}\right) / \Delta r
$$

where $\Delta r$ is the difference in the radial distances of V1 and V2. This gradient is clearly affected by transient disturbances but appears to show no systematic decrease or increase from 1992 through 1994. Although the latitudinal gradient $\left(0.8 \% \mathrm{deg}^{-1}\right.$, $\left.\Delta \Theta-21^{\circ}\right)$ contributes, this intensity gradient is dominated by the radial gradient ( $\sim 4 \% / \mathrm{AU}, \Delta r \sim 12 \mathrm{AU})$. The near constancy of the intensity gradient implies that $C_{f} V / \kappa$, where $C_{f}$ is the Compton-Getting factor (equal to $[2-2 \gamma] / 3$ for nonrelativistic particles, where $\gamma$ is the average power law index of the differential energy spectrum), $V$ is the solar wind velocity, and $\kappa$ is the radial diffusion coefficient, is approximately unchanged while the intensities are increasing by a factor of $\sim 40$. From Figure $2 \mathrm{~b}$ we pick two recent 52-day periods, days 157-209, 1994, and 157-209, 1993, which have V1/V2 intensity gradients near the mean value.

For this study, we use the radial and latitudinal gradients from day 52, 1993, through 365,1994 , representing twelve 52-day periods in Figures $2 \mathrm{c}$ and 2d, in computing the parameters involved in calculating the effective radial locations for the V1 and V2 spacecraft. The average radial gradient, latitudinal gradient, and radial gradient parameter for the twelve periods are $G_{r}=3.92 \pm 0.38 \% \mathrm{AU}^{-1}, G_{\Theta}=0.78 \pm$ $0.18 \% \mathrm{deg}^{-1}$, and $G_{0}=1.96 \pm 0.19$. In Table 1 we show the actual radius and latitude for V1 and V2 for the two sclected time periods along with the calculated effective radial positions used in the model fits.

In the model calculations we fit both the ACR helium and oxygen spectra at V1 and V2. The ACR He shock spectrum is assumed to be a power law in energy per nucleon, $d J / d T=$ $j_{0}\left[T /\left(1 \mathrm{MeV} \text { nucleon }^{-1}\right)\right]^{\gamma}$. The ACR $\mathrm{O}$ shock spectrum is assumed to be a power law with the same index. For () this is an adequate approximation for energies up to $\sim 10 \mathrm{MeV} \mathrm{nu-}$ cleon $^{-1}$. Above $\sim 10 \mathrm{MeV}$ nucleon ${ }^{-1}$ we will later show that the energy spectrum exhibits an approximately exponential roll-off. Similarly, for ACR He this approximation is valid up to $\sim 60 \mathrm{MeV}$ nucleon $^{-1}$. We assume that the diffusion coefficient $\kappa$ (in square centimeters per second) is given by

$$
\kappa=\frac{\kappa_{0} \beta(r / 1 \mathrm{AU})\left(1+\kappa_{S}^{2}\right)(R / 1 \mathrm{GV})^{2}}{1+\left(\kappa_{S} R\right)^{2}} \quad r \gg 1 \mathrm{AU}
$$

where $\kappa_{0}$ is a scaling factor, $\beta$ is particle speed, $r$ is heliocentric radial distance, and $R$ is rigidity. This form for the radial and rigidity dependence of $\kappa$ can be derived from the quasi-lincar formulation of Bieber et al. [1995], in which $\lambda_{\perp}=R_{L} \Omega \tau /(1+$ $\left.\Omega^{2} \tau^{2}\right)$, where $R_{L}$ is the Iarmour radius $\left(\sim R / B_{0}\right), \Omega$ is the gyrofrequency, $\tau$ is the scattering time, and $\Omega \tau=\left[\left(2 R_{L}\right) /\right.$ $\left.\left(3 \lambda_{c}\right)\right]\left(B_{0}^{2} / \delta B_{x}^{2}\right)$. Following Bieber et al., we assume that the coherence length of the magnetic field scales as $r\left(\lambda_{c} \sim r\right)$, that the root-mean-square field luctuations are proportional to the mean field $\left(\delta B_{x}^{2} \sim B_{0}^{2}\right)$, and that $B_{0} \sim r^{-1}$ for $r \gg 1$ AU.

There are six free parameters in the model: the shock location $R_{S}$, the diffusion coefficient scaling factor $\kappa_{0}$, the diffusion coefficient shape factor $\kappa_{S}$, the power law index $\gamma$ of the energy spectrum at the shock, the intensity scaling factor $j_{0}$ of the ACR He shock spectrum, and the ratio of $\mathrm{O}$ to $\mathrm{He}$ at the shock, $\mathrm{O} / \mathrm{He}$. We assume that the solar wind velocity $V$ is constant at $500 \mathrm{~km} \mathrm{~s}^{-1}$, which is close to the average value at V2 of $490 \mathrm{~km} \mathrm{~s}^{-1}$ for day 1, 1993, through 365, 1994 (J. Richardson, private communication, 1995). The fits are sensitive to $\kappa$ and $V$ only in the ratio $\kappa_{0} / V$.

The data and best fit model curves for days 157-209, 1994, are shown in Figures $4 \mathrm{a}$ and $4 \mathrm{~b}$. The fits were made only in the energy regions shown by the solid lines. The $\chi^{2}$ of the sixparameter best fit to the 39 data points in Figure 4 is 13.3. Figure $4 \mathrm{c}$ shows the best fit diffusion coefficient as a function of rigidity.

We investigated the confidence limits for each parameter in two ways. We first estimated the $68 \%$ confidence limits by iteratively changing and fixing the value of one parameter and refitting until we found the parameter value where the $\chi^{2}$ had increased by 1 [see Press et al., 1992]. We did this in turn for all six parameters. The best fit parameter values and the $68 \%$ confidence limits are shown in Table 2 for days 157-209, 1994.

In the second method we account for modeling uncertainties by estimating the uncertainty in the effective radial positions of V1 and V2 using (1) together with the uncertainties in the parameters $G_{0}$ and $G_{\Theta}$. We find that the uncertainties on the effective radial positions were $2 \mathrm{AU}$ for $\mathrm{V} 1$ and $0.5 \mathrm{AU}$ for $\mathrm{V} 2$. We performed two fits, one using the upper limits for the effective radial locations for V1 and V2 and another using the lower limits. The resulting parameters are shown as the model limits in Table 2.

The shock strength $s$ [see, e.g., Potgieter and Moraal, 1988] is related to the spectral index by $s=(2 \gamma-2) /(2 \gamma+1)$. From the values of $\gamma$ in Table 2 , the inferred strength of the shock is $2.63 \pm 0.14$. The shock is therefore not a strong shock $(s=4$; $\gamma=-1$ ), and it may be modified by cosmic rays [see, e.g., Donohue and Zank, 1993]. We estimate that the shock location is $~ 85 \pm 5$ AU during days 157-209, 1994 (see Table 2).

In Figure 5 we show the ACR Hc and O spectra at V1 and V2 for days 157-209, 1993, along with a four-parameter model fit $\left(\chi_{\nu}^{2}=1.1\right)$. We held the shock spectral index fixed at -1.42 and the shock $\mathrm{O} / \mathrm{He}$ ratio fixed at 0.081 , the values from the 
1994 period. The fit indicates that the shock distance has not changed appreciably over the year despite the fact that the peak intensity at V1 has increased by a factor of $\sim 3$. The best fit parameters and limits are shown in Table 3 . This intensity increase is apparently due to a combination of two effects (see Tables 2 and 3): (1) the ratio of the diffusion coefficient at the peak energy of the He spectrum to solar wind velocity has increased by $\sim 50 \%$, and (2) the best fit shock intensity scaling parameter has increased by $\sim 40 \%$, much less than the factor of $\sim 3$ increase in the V1 peak intensity. The relatively constant shock intensity is consistent with quasi-equilibrium conditions for shock accelcration. During the 2-year period around the last solar minimum, Cummings et al. [1994] also found that a constant shock intensity was consistent with observed ACR He
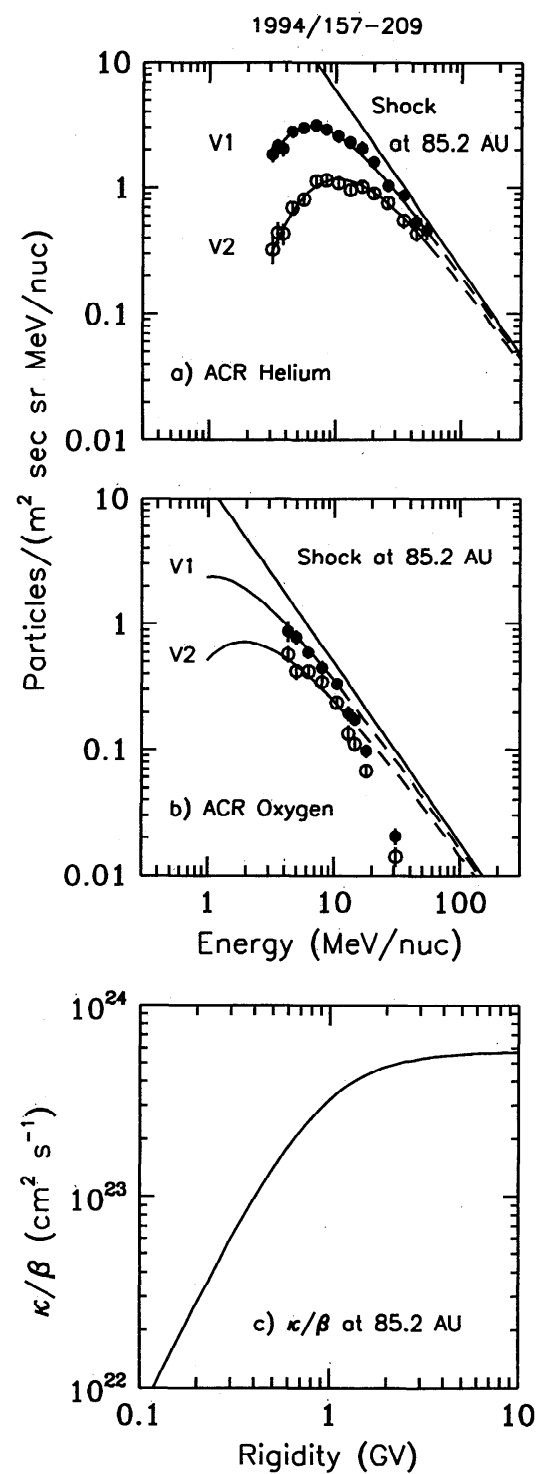

Figure 4. (a) Energy spectra of ACR helium at the positions of V1 and V2 spacecraft for days 157-209, 1994. The curves represent the six-parameter best fit energy spectra at the solar wind termination shock, V1, and V2, as described in the text. (b) Same as Figure 4a except for ACR oxygen. (c) Best fit diffusion coefficient divided by particle velocity versus rigidity. The form of the diffusion coefficient is described in the text.
Table 2. Fit Parameters for Figure 4: Days 157-209, 1994

\begin{tabular}{|c|c|c|c|c|c|}
\hline Parameter ${ }^{\mathrm{a}}$ & Fit Value ${ }^{b}$ & $\begin{array}{l}68 \% \\
\text { Lower } \\
\text { Limit }\end{array}$ & $\begin{array}{l}68 \% \\
\text { Upper } \\
\text { Limit }\end{array}$ & $\begin{array}{c}\text { Model } \\
\text { Lower } \\
\text { Limit }\end{array}$ & $\begin{array}{c}\text { Model } \\
\text { Upper } \\
\text { Limit }\end{array}$ \\
\hline & -1.4 & -1 & -1.34 & -1 & -1.44 \\
\hline$R_{S}$ & & 81.7 & 88.2 & 81.2 & 89.5 \\
\hline$\kappa_{0}\left(\times 10^{21}\right)$ & $3.76( \pm$ & 3.41 & 3.97 & 3.53 & 3.96 \\
\hline$\kappa_{S}$ & 1.13 & 0.98 & 1.28 & 1.16 & 1.12 \\
\hline 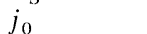 & $158(=$ & 116 & 203 & 153 & 167 \\
\hline $\mathrm{O} / \mathrm{IIe}$ & $0.081( \pm 0.009)$ & 0.073 & 0.090 & 0.083 & 0.080 \\
\hline
\end{tabular}

${ }^{\mathrm{a}} \mathrm{See}$ text for definition of symbols.

${ }^{b}$ Approximate uncertainty in parenthesis derived from the rootmean-square of the statistical $(68 \%)$ uncertainty and the model uncertainty.

and $\mathrm{O}$ intensities that changed by factors of $\sim 5$ and $\sim 8$, respectively.

\section{Roll-Off Energy of Shock Spectrum}

In Figures $4 \mathrm{~b}$ and $5 \mathrm{~b}$ the obscrved ACR oxygen spectra exhibit a roll-off in intensity above $\sim 10-15 \mathrm{MeV}_{\text {nucleon }}{ }^{-1}$. This roll-off is present in other spectra as well as shown in Figure 6 where the V1 ACR O, N, and Ne spectra are displayed for days 157-365, 1994. Note that all three spectra show the roll-off in intensity above $\cdots 10-15 \mathrm{McV}$ nuclcon $^{-1}$. This roll-off in the energy spectra is consistent with that expected in diffusive shock acceleration if the shock has a finite radius of curvature. Such a roll-off is predicted to occur when $V R_{S} / \kappa$ is of the order of unity [see, e.g., Potgieter and Moraal, 1988], where $V$ is the solar wind velocity, $\kappa$ is the diffusion coefficient in the vicinity of the shock, and $R_{S}$ is the shock radius.

Another interpretation of the roll-off in the energy spectrum is due to Jokipii [1990]. In his theory the ACR particles gain their energy by drifting along the shock from the equator to the pole during the $A>0$ portion of the solar cycle (pole to equator during $A<0$ ). The maximum energy gain is set by the electrostatic potential difference between the heliospheric equator and pole and is $\sim 240 \mathrm{MeV}$. Since the ACR particles are singly ionized, the energy spectra of the different ACR ions would be expected to roll off above $\sim 240 \mathrm{MeV}$.

In Figure 7 we plot the V1 differential energy spectra of ACR $\mathrm{H}, \mathrm{He}, \mathrm{N}, \mathrm{O}$, and $\mathrm{Ne}$ for days 157-365, 1994, as a function of energy instead of energy per nucleon. The intensities have been normalized before plotting by the factors shown in the figure. At low energies the effect of solar modulation causes the $\mathrm{H}$ and $\mathrm{He}$ spectra to peak at different energies. However, the agreement of the different spectra is good near the break which occurs at $\sim 170-240 \mathrm{MeV}$, in rough agrecment with the shock drift limit of Jokipii [1990]. Wc do not feel that we can distinguish experimentally between the two possible interpretations of the roll-off in the shock spectra.

\section{Latitudinal Gradient Along Shock}

The shock spectra of ACR He and O in Figures $4 a$ and $4 b$ are the spectra expected at the shock in the heliographic equator for days 157-209, 1994. Likewise, the shock spectra derived by Cummings et al. [1994] for days 105-313, 1987, are the spectra expected at the shock in the heliographic equator. For the 1987 period the intensity of ACR He at the shock in the energy range $31-57 \mathrm{MeV}$ nucleon ${ }^{-1}$ was determined to be 4.3 


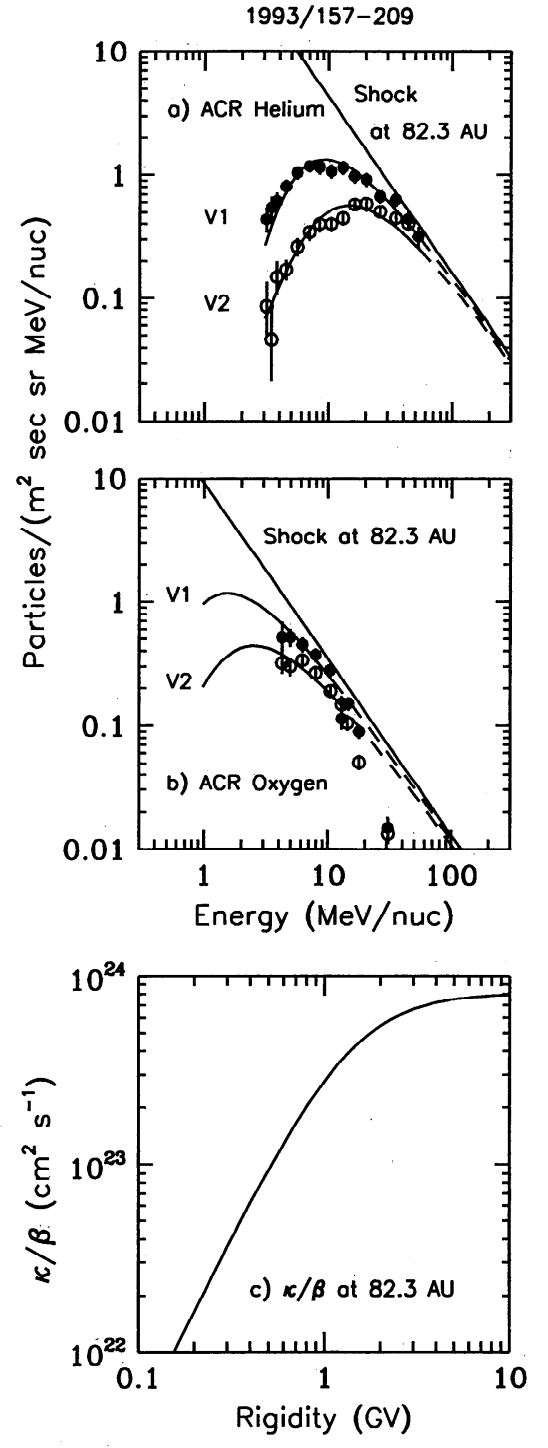

Figure 5. Same as Figure 4 except the period is days 157209,1993 , the shock power law spectral index is fixed at -1.42 , and the $\mathrm{O} / \mathrm{He}$ ratio is fixed at 0.081 .

$\left(\mathrm{m}^{2} \text { s sr MeV/nucleon }\right)^{-1}$. From Figure 4a the ACR He shock intensity during days 157-209, 1994, in this same energy range is $0.77\left(\mathrm{~m}^{2} \mathrm{~s} \text { sr MeV/nucleon }\right)^{-1}$. Thus the intensity at the shock at the heliographic equator in 1994 is a factor of $\sim 5.5$

Table 3. Fit Parameters for Figure 5: Days 157-209, 1993

\begin{tabular}{|c|c|c|c|c|c|}
\hline Parameter $^{2}$ & Fit Value ${ }^{b}$ & $\begin{array}{l}68 \% \\
\text { Lower } \\
\text { Limit }\end{array}$ & $\begin{array}{l}68 \% \\
\text { Upper } \\
\text { Limit }\end{array}$ & $\begin{array}{c}\text { Model } \\
\text { Lower } \\
\text { Limit }\end{array}$ & $\begin{array}{l}\text { Model } \\
\text { Upper } \\
\text { Limit }\end{array}$ \\
\hline 7 & $1.42^{\mathrm{c}}$ & 0 & 0 & 0 & 0 \\
\hline$R_{S}, \mathrm{AU}$ & $82.3( \pm 4.1)$ & 79.7 & 84.1 & 78.1 & 85.1 \\
\hline$\kappa_{0} \times 10^{21}$ & $3.35( \pm 0.26)$ & 3.15 & 3.56 & 3.18 & 3.51 \\
\hline$\kappa_{S}$ & $0.72( \pm 0.10)$ & 0.62 & 0.81 & 0.73 & 0.6 \\
\hline$j_{0}$ & $113( \pm 9)$ & 104 & 122 & 111 & 111 \\
\hline $\mathrm{O} / \mathrm{He}$ & $0.081^{\mathrm{c}}$ & 0 & 0 & 0 & 0 \\
\hline
\end{tabular}

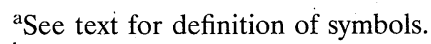

'Approximate uncertainty in parenthesis derived from the rootmean-square of the statistical (68\%) uncertainty and the model uncertainty.

${ }^{\mathrm{c}}$ Held fixed.

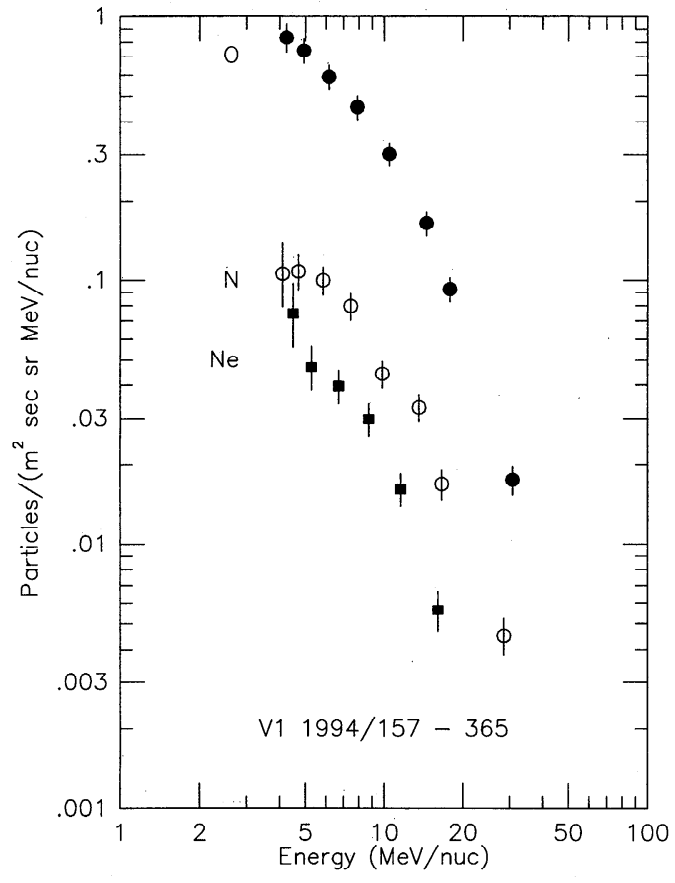

Figure 6. Energy spectra of ACR $\mathrm{O}, \mathrm{N}$, and $\mathrm{Ne}$ for days 157-365, 1994. Small (<10\%) corrections for GCRs have been made before plotting.

smaller than it was in 1987. This difference can be understood in the context of shock drift acceleration [Jokipii, 1990] that causes the intensity to vary along the shock from the equator to the pole, the intensity being a maximum at the pole during the 1994 period $(A>0)$ and a maximum at the equator during $1987(A<0)$. Interpreted in this way, the factor of 5.5 difference observed in the 1994 shock intensity compared to the 1987 shock intensity could correspond to the latitudinal difference in the intensity at the equator and the pole in either of the years 1994 or 1987 . This would imply a latitudinal gradient along the shock of $1.9 \% \mathrm{deg}^{-1}$ from the equator to the pole during days 157-209, 1994, in qualitative agreement with shock drift acceleration. In Table 4 we show this value of the

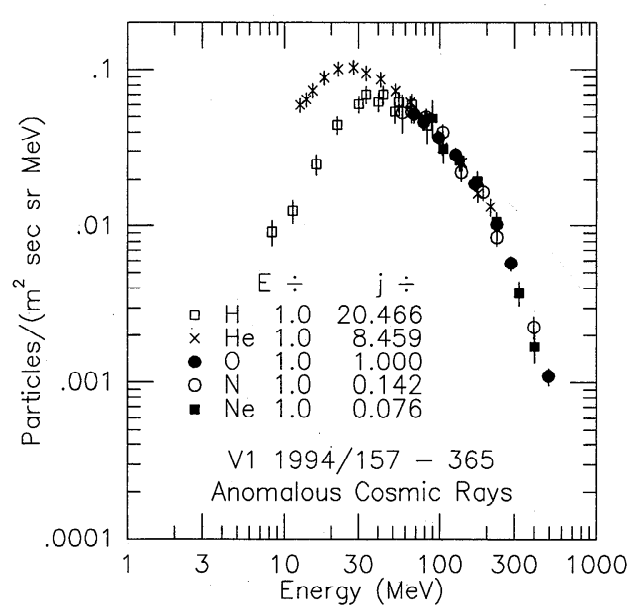

Figure 7. Composite V1 energy spectrum of $\mathrm{O}$ for days 157 365,1994 , plotted as a function of total energy. The energy spectra of the different individual ions are normalized to $\mathrm{O}$ by the factors shown in the figure. 
latitudinal gradient along with several other recent determinations for the latitudinal gradient of ACRs, most of which are based on comparisons of Ulysses measurements at high latitude with measurements near the ecliptic plane. In general, there is a correspondence of these measurements with the implied latitudinal gradient along the shock.

\section{Energy Density of ACRs}

The energy density of the ACR He shock spectrum in Figure 4a above an injection velocity of $2 V_{S W}\left(5.2 \mathrm{keV}\right.$ nucleon ${ }^{1}$ for $V_{S W}=500 \mathrm{~km} \mathrm{~s}^{-1}$ ) is $\sim 0.005 \mathrm{eV} \mathrm{cm}^{-3}$. The corresponding value for ACR $\mathrm{O}$ in Figure $4 \mathrm{~b}$ is $-0.001 \mathrm{eV} \mathrm{cm}^{-3}$. We can estimate the ACR H shock spectrum by scaling from the ACR He shock spectrum. Cummings et al. [1984] found that the ACR energy spectra of the different elements scale to each other with linear factors in energy and intensity if the diffusion coefficient is proportional to $\beta R^{\alpha}$. The peak energies of the ACR $\mathrm{H}$ and $\mathrm{He}$ spectra are in the rigidity range $(\sim 0.3-0.4$ $\mathrm{GV}$ ) where $\alpha$ is $\sim 2$ (see Figures $4 \mathrm{c}$ and $5 \mathrm{c}$ ). This implies an energy scaling factor of 6.3 [see Cummings et al., 1984]. The intensity scaling factor is $0.40 \pm 0.02$ from Cummings and Stone [1995] for a period only slightly different from the one shown in Figure 4. Using these factors and the He spectrum from Figure 4a, we estimate that the power law portion of the ACR $\mathrm{H}$ shock spectrum for days $157-209,1994$, is given by $j_{H}=868$ $E^{-1.42}\left(\mathrm{~m}^{2} \mathrm{~s} \text { sr } \mathrm{MeV}\right)^{-1}$. This implies an energy density of ACR $\mathrm{H}$ at the shock of $0.009 \mathrm{eV} \mathrm{cm}^{-3}$, yielding a total $\Lambda \mathrm{CR}$ energy density of $0.015 \pm 0.002 \mathrm{eV} \mathrm{cm}^{-3}$. The estimated uncertainty includes uncertainty in $\gamma$ from Table 2 and the uncertainty in scaling the $\mathrm{He}$ shock spectrum to the $\mathrm{H}$ shock spectrum. For comparison, the energy density of the solar wind at low heliographic latitudes at $85 \mathrm{AU}$ is $\sim 1.2 \mathrm{eV} \mathrm{cm}^{-3}$, as scaled from $\sim 16 \mathrm{eV} \mathrm{cm}^{-3}$ at $23 \mathrm{AU}$ (derived from Figure 1 of Grzedielski and Lazarus [1993]). Thus the ACR energy density is only $\sim 1 \%$ that of the solar wind.

This ACR energy density is similar to that derived for the 1987 period by Cummings et al. [1994]. However, Cummings et al. [1994] assumed a strong shock with a power law index at low energies of -1 . The modulation effects were stronger in the 1987 spectrum at $\sim 23$ AU than they are in the 1994 spectrum at $-56 \mathrm{AU}$, and the peak energy of the $1987 \mathrm{ACR} \mathrm{Hc}$ spectrum was $\sim 20-25 \mathrm{MeV}$ nucleon ${ }^{-1}$ compared to $\sim 6 \mathrm{MeV}$ nucleon $^{-1}$ at V1 in 1994 (see Figure 4a). Consequently, in the

Table 4. Latitudinal Gradients of ACRs

\begin{tabular}{|c|c|c|c|c|c|}
\hline & ACR & $\begin{array}{c}E, \\
\mathrm{MeV}^{-1} \\
\text { nucleon }^{-1}\end{array}$ & $\begin{array}{c}r, \\
\mathrm{AU}\end{array}$ & $\begin{array}{c}\theta, \\
\text { deg }\end{array}$ & $\begin{array}{c}G_{\theta} \\
\% \\
\mathrm{deg}^{-1}\end{array}$ \\
\hline Polar/equatorial shock & $\mathrm{He}$ & $31-57$ & $67-85$ & 90 & 1.9 \\
\hline ULY-IMP-V1-V2-P10 & $\mathrm{O}$ & 10 & 2.3 & -77 & $2.1 \pm 0.6$ \\
\hline \multirow[t]{3}{*}{ ULY-SAMPEX } & $\mathrm{O}$ & $8-12$ & 2.3 & -80 & $2.12 \pm 0.27$ \\
\hline & $\mathrm{O}$ & $12-16$ & & & $1.75 \pm 0.38$ \\
\hline & $\mathrm{He}$ & $11-20$ & & & $0.10 \pm 0.18$ \\
\hline ULY-IMP $8^{\mathrm{c}}$ & $\mathrm{He}^{\mathrm{c}}$ & $30-70$ & $\sim 2$ & -80 & $0.57 \pm 0.10^{\mathrm{c}}$ \\
\hline ULY-IMP-V1-V2-P10 & $\mathrm{O}$ & 10 & 4.2 & -41 & $1.3 \pm 0.3$ \\
\hline $\mathrm{V} 1-\mathrm{V} 2-\mathrm{P} 10^{\mathrm{e}}$ & $\mathrm{He}$ & $10-22$ & 55 & 32 & $0.8 \pm 0.1$ \\
\hline
\end{tabular}

${ }^{\mathrm{a} C}$ Cummings et al. [1995b].

${ }^{\mathrm{b}}$ Trattner et al. [1995].

Simpson et al. [1995] (includes GCR flux, so is lower limit for ACR gradient).

${ }^{\mathrm{d}}$ Cummings et al. [1995a].

${ }^{\mathrm{e}}$ Stone et al. [1995b].

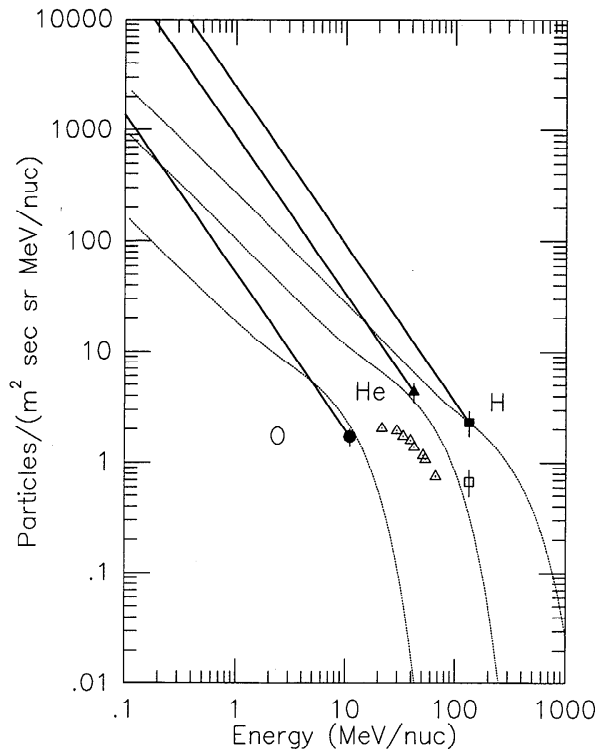

Figure 8. Same as Figure 5b from Cummings et al. [1994] showing the energy spectra of ACR $\mathrm{O}, \mathrm{He}$, and $\mathrm{H}$ for days 105-313, 1987. The open points are observations from V2. The solid points are the deduced intensities at the termination shock. Three power law lines have been added, normalized to the shock intensities, with power law index of -1.42 to indicate a possibly more realistic encrgy dependence of the shock spectra.

1987 observation there was very little information on the power law index of the shock spectrum. In Figure 8 we reproduce Figure 5b from Cummings et al. [1994] with the addition of lines indicating possible shock spectra with power law index of -1.42 in 1987. Assuming that this index was -1.42 in 1987 results in somewhat higher estimates of the energy density of ACRs at the shock than were derived by Cummings et al. [1994]. In Table 5 we show the estimated energy density for the 1994 period along with the old and new estimates for 1987. We also compare to the estimated energy density of the solar wind at the location of the shock. If we assume that the 1987 measurements with index -1.42 correspond to the maximum ACR intensity at the shock, then the energy density of ACRs is $\sim 5 \%$ of the solar wind energy density in the region with maximum source intensity in 1994.

\section{Injection Efficiency of Pickup Ions at Shock}

To estimate the efficiency $r_{i}$ for the injection of pickup ions into the acceleration process, we follow the calculations of Lee [1983], from which it can be shown that the accelerated spectrum is given in units of $\left(\mathrm{cm}^{2} \mathrm{~s} \text { sr } \mathrm{MeV} / \text { nucleon }\right)^{-1}$ by

Table 5. Estimated Energy Densities

\begin{tabular}{ccccc}
\hline Period & $\begin{array}{c}R_{S}, \\
\mathrm{AU}\end{array}$ & $\gamma$ & $\begin{array}{c}\varepsilon_{\mathrm{ACR}}{ }^{\mathrm{a}} \\
\mathrm{eV} \mathrm{cm}\end{array}$ & $\begin{array}{c}\varepsilon_{\mathrm{ACR}} / \varepsilon_{\mathrm{SW}}, \\
\%\end{array}$ \\
\hline 1994 & 85 & -1.42 & 0.015 & 1.3 \\
1987 & 67 & $-1.00^{\mathrm{b}}$ & 0.028 & 1.5 \\
1987 & 85 & $-1.42^{\mathrm{c}}$ & 0.058 & 4.8 \\
\hline
\end{tabular}

a Assumes spectral slope extends down to $5.2 \mathrm{keV}$ nucleon ${ }^{-1}$.

bStrong shock assumed $(s=4)$.

'Shock $s=2.63$ assumed. 


$$
j=p^{2} f(p)=\frac{\nu r_{i} F_{i}}{4 \pi} E_{0 i}^{(\nu-4) / 2} E^{(-\nu+2) / 2}
$$

where $\nu \equiv 3 /\left(1-\rho_{u} / \rho_{d}\right)=4.84$ for a downstream/upstream density ratio of $2.63, F_{i}$ (in square centimeters per second) is the pickup ion flux at the shock, and $E_{0 i}$ is the injection energy corresponding to $2 V_{S W}$, taken here to be $5.2 \times 10^{-3} \mathrm{MeV}$ nucleon $^{-1}$. The pickup ion fluxes at the shock can be estimated from the observations of Gloeckler et al. [1993], who inferred the density of neutral $\mathrm{H}$ and $\mathrm{He}$ in the outer heliosphere as $0.077 \pm 0.015$ and $0.013 \pm 0.003 \mathrm{~cm}^{3}$, respectively. Using these values, the model of Vasyliunas and Siscoe [1976] for the distribution of these neutrals throughout the heliospherc, and the long-term ionization rates at $1 \mathrm{AU}$ of $8.0 \times 10^{-7} \mathrm{~s}^{-1}$ for $\mathrm{H}$ and $1.0 \times 10^{-7} \mathrm{~s}^{-1}$ for He [Gloeckler et al., 1993], we estimate that the fluxes of $\mathrm{H}$ and $\mathrm{He}$ pickup ions at the nose of the heliosphere at $85 \mathrm{AU}$ are $8500 \pm 1700 \mathrm{~cm}^{-7} \mathrm{~s}^{-1}$ and $220 \pm 50$ $\mathrm{cm}^{-2} \mathrm{~s}^{-1}$, respectively.

Using these pickup ion fluxes, the calculated ACR He spectrum from Lee [1983] of $j_{H e}=9.28 \times 10^{4} r_{i} E^{-1.42}\left(\mathrm{~m}^{2} \mathrm{~s} \mathrm{sr}\right.$ $\mathrm{MeV} /$ nucleon $)^{-1}$ can be compared with the observed $j_{H e}=$ $158 E^{-1.42}\left(\mathrm{~m}^{2} \mathrm{~s} \mathrm{sr} \mathrm{MeV} / \text { nucleon }\right)^{-1}$ to yield $r_{i}\left(\mathrm{He}^{+}\right)-0.0017$ \pm 0.0008 . The estimated uncertainty includes the uncertainty in $\gamma$ from Table 2 and the uncertainty in the pickup ion flux $F_{i}$. For $\mathrm{H}$ the calculated spectrum is $j_{I I}=3.59 \times 10^{6} r_{i} E^{-1.42}$ $\left(\mathrm{m}^{2} \mathrm{~s} \text { sr MeV}\right)^{-1}$ and the "observed" spectrum is $j_{H}=$ $868 E^{-1.42}\left(\mathrm{~m}^{2} \mathrm{~s} \text { si } \mathrm{MeV}\right)^{-1}$. We thus find that the injection efficiency for $\mathrm{H}$ is $r_{i}\left(\mathrm{H}^{+}\right)=0.00024 \pm 0.00011$, where we include an additional uncertainty due to the scaling of the $\mathrm{He}$ shock spectrum to the $\mathrm{H}$ shock spectrum. The ratio of the injection efficiencies of $\mathrm{He}^{+}$and $\mathrm{H}^{+}$is relatively better determined than the absolute injection efficiencies of either because the spectral index uncertainty is removed in the ratio. We estimate that the ratio of the efficiency of injection of $\mathrm{He}^{+}$to $\mathrm{H}^{+}$pickup ions into the acceleration process is $7.0 \pm 1.6$, where we have used the $\mathrm{He}$ to $\mathrm{H}$ scaling uncertainty in the shock spectra $(16 \%)$ and the uncertainty on the ratio of the neutral densities of $\mathrm{H}$ to $\mathrm{He}$ in the outer heliosphere from Gloeckler et al. [1993] (17\%) in estimating the uncertainty in the ratio. The observed preferential injection for $\mathrm{He}^{+}$compared to $\mathrm{H}^{+}$is qualitatively consistent with Monte Carlo studies of shock acceleration by Ellison et al. [1981].

\section{Discussion}

Our estimates of energy spectra and shock location correspond to the upwind termination shock in the heliographic equator, since both V1 and V2 are headed outward in that general direction. Even if the termination shock is elongated in the downwind direction [see, e.g., Seuss et al., 1987], the nose region of the upwind shock should be reasonably approximated by a spherically symmetric diffusion model. In the downwind direction, azimuthal diffusion parallel to the magnetic field will become increasingly important with increasing shock elongation, tending to reduce upwind/downwind asymmetries in the ACR intensities.

We will continue to monitor the evolution of the energy spectra of ACRs at V1 and V2 and look for further clues to the strength of the shock and its location. The energy of the peak intensity should continue to decrease as the spacecraft approaches the shock. As the modulation lessens, the convergence of the V1 and V2 spectra should continue to lower energies, and we will obtain a better estimate of the shock spectrum spectral index. As solar activity increases from the solar minimum conditions in 1998, the shock may move rapidly inward [see, e.g., Whang and Burlaga, 1993] from its position at $\sim 85$ AU and perhaps overrun the positions of the V1 and P10 spacecraft, which will both be at $\sim 72 \Lambda \mathrm{U}$, some $5 \Lambda \mathrm{U}$ beyond the estimated location of the shock 11 years earlier [Cummings et al., 1994].

Acknowledgments. We are grateful to J. T. Hoeksema for providing the tilt observations prior to publication. We thank J. Richardson and J. Belcher for providing the Voyager 2 solar wind speed data. This work was supported by NASA under contract NAS7-918.

The Editor thanks D. T. Hall and two other referees for their assistance in evaluating this paper.

\section{References}

Adams, J. H., Jr., et al., The charge state of the anomalous component of cosmic rays, Astrophys. J. Lett., 375, L45, 1991.

Belcher, J. W., A. J. Lazarus, R. L. McNutt Jr., and G. S. Gordon Jr., Solar wind conditions in the outer heliosphere and the distance to the termination shock, J. Geophys. Res., 98, 15,177, 1993.

Bieber, J. W., R. A. Burger, and W. H. Matthaeus, The diffusion tensor throughout the heliosphere, Proc. Int. Conf. Cosmic Rays, 24th, SH, 694, 1995.

Christian, E. R., A. C. Cummings, and E. C. Stone, Evidence for anomalous cosmic ray hydrogen, Astrophys. J. Lett., 334, L77, 1988.

Christian, E. K., A. C. Cummings, and E. C. Stone, Observations of anomalous cosmic ray hydrogen from the Voyager spacecraft, $A s$ trophys. J. Lett., 446, L105, 1995.

Cummings, A. C., and E. C. Stone, Composition, gradients, and temporal variations of the anomalous cosmic-ray component, in Solar Wind Six, Tech. Note NCAR/TN-306+Proc, p. 599, Natl. Cent. for Atmos. Res., Boulder, Colo., 1988.

Cummings, A. C., and E. C. Stone, Elemental composition of the very local interstellar medium as deduced from observations of anomalous cosmic rays, Proc. Int. Conf. Cosmic Rays, 21st, 6, 202, 1990.

Cummings, A. C., and E. C. Stone, Elemental composition of the anomalous cosmic ray component, Proc. Int. Conf. Cosmic Rays, 24th, SII, 497, 1995.

Cummings, A. C., E. C. Stone, and W. R. Webber, Evidence that the anomalous cosmic-ray component is singly-ionized, Astrophys. J. Lett., 287, L99, 1984.

Cummings, A. C., E. C. Stone, and W. R. Webber, Latitudinal and radial gradients of anomalous and galactic cosmic rays in the outer heliosphere, Geophys. Res. Lett., 14, 174, 1987.

Cummings, A. C., E. C. Stone, and W. R. Webber, Distance to the solar wind termination shock and the source flux of anomalous cosmic rays during 1986-1988, J. Geophys. Res., 99, 11,547, 1994.

Cummings, A. C., et al., Anomalous cosmic ray oxygen gradients throughout the heliosphere, Geophys. Res. Lett., 22, 341, 1995a.

Cummings, A. C., et al., Radial and latitudinal gradients of anomalous cosmic ray oxygen throughout the heliosphere, Proc. Int. Conf. Cosmic Rays, 24th, SH, 800, 1995b.

Donohue, D. J., and G. P. Zank, Steady state and dynamical structure of a cosmic-ray-modified termination shock, J. Geophys. Res., 98, $19,005,1993$.

Ellison, D. C., F. C. Jones, and D. Eichler, Monte Carlo simulation of collisionless shocks showing preferential acceleration of high $A / Z$ particles, J. Geophys., 50, 110, 1981.

Fisk, L., B. Kozlovsky, and R. Ramaty, An interpretation of the observed oxygen and nitrogen enhancements in low-energy cosmic rays, Astrophys. J. Lett., 190, L35, 1974.

Garcia-Munoz, M., G. M. Mason, and J. A. Simpson, A new test for solar modulation theory: The 1972 May-July low-energy galactic cosmic ray proton and helium spectra, Astrophys. J. Letl., 182, L81, 1973.

Gloeckler, G., J. Geiss, H. Balsinger, L. A. Fisk, A. B. Galvin, F. M. Ipavich, K. W. Ogilvie, R. von Steiger, and B. Wilken, Detection of interstellar pick-up hydrogen in the solar system, Science, 261, 70, 1993.

Grzedielski, S., and A. J. Lazarus, 2- to $3-\mathrm{kHz}$ continuum emissions as possible indications of global heliospheric "breathing," J. Geophys. Res., 98, 5551, 1993. 
Gurnett, D. A., W. S. Kurth, S. C. Allendorf, and R. L. Poynter, Radio emission from the heliopause triggered by an interplanetary shock, Science, 262, 199, 1993.

Hall, D. T., D. E. Shemansky, D. L. Judge, P. Gangopadhyay, and M. A. Gruntman, Heliospheric hydrogen beyond 15 AU: Evidence for a termination shock, J. Geophys. Res., 98, 15,221, 1993.

Hoeksema, J. T., Large-scale structure of the heliospheric magnetic field: 1976-1991, in Solar Wind Seven, COSPAR Colloq. Ser., vol. 3, edited by E. Marsch and R. Schwenn, p. 191, Pergamon, New York, 1992.

Hovestadt, D., O. Vollmer, G. Gloeckler, and C. Y. Fan, Differential energy spectra of low-energy ( $8.5 \mathrm{MeV}$ per nucleon) heavy cosmic rays during solar quiet times, Phys. Rev. Lett., 31, 650, 1973.

Ip, W.-H., On using the ACR to probe the LISM/heliosphere interface, in Physics of the Outer Heliosphere, COSPAR Colloq. Ser., vol. 1, edited by S. Grzedielski and D. E. Page, p. 307, Pergamon, New York, 1990.

Jokipii, J. R., The anomalous component of cosmic rays, in Physics of the Outer Heliosphere, COSPAR Colloq. Ser., vol. 1, edited by S. Grzedielski and D. E. Page, p. 169, Pergamon, New York, 1990.

Jokipii, J. R., E. H. Levy, and W. R. Hubbard, Effects of particle drift on cosmic-ray transport, I, General properties, applications to solar modulation, Astrophys. J., 213, 861, 1977.

Klecker, B., M. C. McNab, J. B. Blake, D. C. Hamilton, D. Hovestadt, H. Kästle, M. D. Looper, G. M. Mason, J. E. Mazur, and M. Scholer, Charge-state of anomalous cosmic-ray nitrogen, oxygen, and neonSAMPEX observations, Astrophys. J. Lett., 442, L69, 1995.

Lallement, R., J.-L. Bertaux, and J. T. Clarke, Deceleration of interstellar hydrogen at the heliospheric interface, Science, 260, 1095, 1993.

Lee, M. A., Coupled hydromagnetic wave excitation and ion acceleration at interplanetary traveling shocks, J. Geophys. Res., 88, 6109, 1983.

Le Roux, J. A., and M. S. Potgieter, A time-dependent drift model for the long-tcrm modulation of cosmic rays with special reference to asymmetries with respect to the solar minimum of 1987, Astrophys. J., 361, 275, 1990.

McDonald, F. B., B. J. Teegarden, J. H. Trainor, and W. R. Webber, The anomalous abundance of cosmic-ray nitrogen and oxygen nuclei at low energies, Astrophys. J. Lett., 187, L105, 1974.

McDonald, F. B., A. Barnes, L. F. Burlaga, P. Gazis, J. Mihalov, and R. S. Selesnick, Effects of the intense solar activity of March/June 1991 observed in the outer heliosphere, J. Geophys. Res., $99,14,705$, 1994.

McDonald, F. B., A. Lukasiak, and W. R. Webber, Pioneer 10 and Voyager 1 observations of anomalous cosmic ray hydrogen in the outer heliosphere, Astrophys. J. Lett., 446, L101, 1995.

Pesses, M. E., J. R. Jokipii, and D. Eichler, Cosmic ray drift, shock wave acceleration, and the anomalous component of cosmic rays, Astrophys. J. Lett., 246, L85, 1981.

Potgieter, M. S., and H. Moraal, Accclcration of cosmic rays in the solar wind termination shock, 1 , A steady state technique in a spherically symmetric model, Astrophys. J., 330, 445, 1988.

Press, W. H., S. A. Teukolsky, W. T. Vettering, and B. P. Flannery, Numerical Recipes in C: The Art of Scientific Computing, 2nd ed., p. 551, Cambridge Univ. Press, New York, 1992.

Seuss, S. T., D. H. Hathaway, and A. J. Dessler, Asymmetry of the heliosphere, Geophys. Res. Lett., 14, 977, 1987.

Simpson, J. A., J. J. Connell, C. Lopate, R. B. McKibben, and M. Zhang, The latitude gradients of galactic cosmic rays and anomalous helium fluxes measured on Ulysses from the Sun's south polar region to the equator, Geophys. Res. Lett., 22, 3337, 1995.

Singh, R. K., M. Banashree, N. Durgaprasad, S. Biswas, M. N. Vahia, J. S. Yadav, A. Dutta, and J. N. Goswami, Ionization states of the anomalous cosmic rays, Astrophys. J., 374, 753, 1991.

Stone, E. C., R. E. Vogt, F. B. McDonald, B. J. Tecgarden, J. H. Trainor, J. R. Jokipii, and W. R. Webber, Cosmic ray investigation for the Voyager missions: Energetic particle studies in the outer heliosphere and beyond, Space Sci. Rev., 21, 355, 1977.

Stone, E. C., A. C. Cummings, and W. R. Webber, Evolution of the energy spectra of anomalous cosmic rays in the outer heliosphere and the distance to the solar wind termination shock, Proc. Int. Conf. Cosmic Rays, 24th, SH, 792, 1995a.

Stone, E. C., A. C. Cummings, and W. R. Webber, Radial and latitudinal gradients of anomalous cosmic rays in the outer heliosphere, Proc. Int. Conf. Cosmic Rays, 24th, SH, 796, 1995b.

Trattner, K. J., R. G. Marsden, T. T. Sanderson, K.-P. Wenzel, B. Klecker, and D. Hovestadt, The anomalous component of cosmic rays: Oxygen latitudinal gradient, Geophys. Res. Lett., 22, 337, 1995.

Vasyliunas, V. M., and G. L. Siscoe, On the flux and the energy spcctrum of intcrstcllar ions in the solar systcm, J. Geophys. Res., 81, 1247, 1976.

Whang, Y. C., and L. F. Burlaga, Termination shock: Solar cycle variations of location and speed, J. Geophys. Res., 98, 15,221, 1993.

Zank, G. P., I. H. Cairns, D. J. Donohue, and W. H. Matthaeus, Radio emissions and the heliospheric termination shock, J. Geophys. Res., 99, 14,729, 1994

A. C. Cummings and E. C. Stone, Space Radiation Laboratory, Mail Code 220-47, California Institute of Technology, Pasadena, CA 91125. (e-mail: ace@citsrl.caltech.edu; ecs@citsrl.caltech.edu)

W. R. Webber, Department of Astronomy, New Mexico State University, Las Cruces, NM 88003. (e-mail: bwebber@nmsu.edu)

(Received August 17, 1995; revised January 25, 1996; acceptcd January 29, 1996.) 